\title{
„Gendergerechtes Sprechen“" als Evidenz für Sprachkontakt: Der Fall des Sorbischen
}

$\mathrm{Zu}$ s a m m e n fas s u ng: In einer kleinen Korpusstudie mit Texten aus der niederund obersorbischen Publizistik wird die Verbreitung gendergerechter sprachlicher Ausdrucksmittel untersucht. Im Wege der close-reading-Methode wurden die Strategien des Splittings (Beidnennung) sowie verschiedene Typen genusneutraler Konstruktionen geprüft. Allgemein sind explizite genderlinguistische Strukturen wie die Beidnennung nicht sehr häufig vertreten. Sie sind eher als selten gebrauchte emblematische sprachliche Symbole zu verstehen. Das generische Maskulinum ist in den Texten weit verbreitet. Lexikalisch sind genderlinguistische Ausdrucksmittel als Übernahmen aus dem Deutschen gut erkennbar.

Schlüsselwörter: Sorbisch; Genderlinguistik; Sprachkontakt; Minderheitensprachen; Zeitungssprache

\section{Genderlinguistische Grundlagen der vorliegenden Untersuchung}

In vielen west- und mitteleuropäischen Ländern ist die Durchsetzung einer Agenda des gendergerechten Sprechens ${ }^{1}$ in eine Phase getreten, die auch für

${ }^{1}$ Dieser Begriff wird hier zur Benennung der Gesamtheit gendersprachlicher Aktivitäten in der Praxis verwendet. Er bezieht sich auf sprachpolitische Vorschläge, die durchaus nicht nur von Linguisten gemacht werden, sondern vielfach von feministischen 
die deskriptiv orientierte Linguistik von Interesse ist. Vor allem funktionale und strukturelle Aspekte des aktuellen Sprachwandels lassen sich am Beispiel des genderorientierten Sprachaktivismus fortlaufend in früher kaum geahnten Konstellationen beobachten.

Die vielfältigen Erscheinungsformen dieser soziokulturellen Bewegung in den pluralistischen Gesellschaften der Gegenwart werden auf drei grundlegende Motivationen zurückgeführt, die in einer chronologischen Abfolge gesellschaftspolitischer Diskussionen stehen (s. Motschenbacher 2015: 52-55):

1) Bestrebungen, „Frauen in der Sprache besser sichtbar zu machen“. Diese Einstellung kann auch als eine Ambition galanter Höflichkeit aufgefasst werden, die traditionelle Geschlechterrollen verstärkt und feministischen Interessen eher entgegensteht.

2) Versuche, die Interessen von Frauen und Männern in der Rede gleichberechtigt abzubilden. Geschlechterunterschiede sollen konsequent versprachlicht werden; das eine Geschlecht ist dem anderen nicht stillschweigend zu subsumieren. Auf die Problematik, dass auf diese Weise Themenbereiche sexualisiert werden, in denen Geschlechtsunterschiede sachlich irrelevant sind, wurde bereits frühzeitig hingewiesen (Leiss 1994).

3) Die Auflösung fester Geschlechterrollen. Das soziale Geschlecht (Gender) ist flexibel und „verhandelbar“. Insofern sollte es auch nicht durch Lexik und Grammatik festgeschrieben sein. Alle sprachlichen Formen sind zu vermeiden, die eine Geschlechterbinarität zum Ausdruck bringen.

Jede dieser Thesen stellt spezifische Ansprüche an die Ausgestaltung der gendergerechten Rede: z. B. können Bezeichnungen weiblicher Personen vor den Bezeichnungen männlicher Personen angeordnet werden (1), das generische Maskulinum ist überhaupt durch Beidnennung zu ersetzen (2), binäre genusspezifische Wortpaare sind durch genderneutrale Abstrakta zu umschreiben (3). So ergeben sich gewisse Widersprüchlichkeiten - z. B. dass sowohl die Beidnennung der Geschlechter als auch die Neutralisierung von Geschlechterkontrasten in das Interessenspektrum des Genderaktivismus

Aktivisten. Ich möchte den suggestiven Begriff Gendergerechtigkeit nicht als Wertung dieser Ansätze verstanden wissen. In meinem Text verzichte ich bewusst auf die Verwendung derjenigen gendergerechten Ausdrucksmittel, die Thema dieses Aufsatzes sind. 
fällt. Das ist aber aus der zeitgeschichtlichen Entwicklung dieser Forderungen, die sich über mehrere Jahrzehnte erstreckt, gut erklärlich und sollte nicht als Argument gegen die Akzeptanz genderlinguistischer Ansätze benutzt werden. Auch der Umstand, dass es Vertretern der Genderlinguistik mitunter selbst schwer fällt, sich in Paradigmen der strukturalistischen Linguistik einzufügen (s. Scheller-Boltz 2015: 15, diskutiert bei Menzel 2021: 106 f.), sollte nicht dazu dienen, einen unüberbrückbaren Gegensatz zwischen System- und Genderlinguistik zu konstruieren. Sicherlich verstehen sich einige Protagonisten der gendergerechten Sprache mehr als sprachpolitische Aktivisten denn als einer deskriptiven Linguistik verpflichtete Grundlagenforscher. Ihre Arbeiten können aber auf vielfältige Weise in soziolinguistischen Studien genutzt werden.

Eine Herausforderung für die Sprachwissenschaft der Gegenwart, gerade wenn sie auch an diachronem Wandel interessiert ist, besteht darin, den traditionellen Gegensatz zwischen der Beschreibung von ,endogenen“ (grammatischen, strukturellen) und „exogenen“ (soziolinguistischen, sprachkontaktbedingten) Einflussfaktoren zu überwinden (vgl. bereits Anttila 1989: 391). Genderpolitische Sprachplanung bietet analytisch hochinteressante Szenarien des exogenen, durch eine relativ kleine gesellschaftliche Schicht a u foktroyierten Sprachwandels. In der Vergangenheit wurde die Durchsetzbarkeit derartiger sprachplanerischer Eingriffe von oben in der breiten Masse der Sprecher mit guten Gründen in Abrede gestellt. Das nun angebrochene Medienzeitalter bietet hier allerdings Möglichkeiten, die zuvor undenkbar gewesen wären. Wenn sich Vertreter der Genderlinguistik als strategisch handelnde feministische Aktivisten erweisen, ${ }^{2}$ sind die Details ihres Vorgehens von erheblichem Interesse sowohl für allgemeine soziolinguistische Fragestellungen als auch für funktionale Analysen des Sprachsystems. Immerhin stellen sie Forderungen, die dem kommunikativen Interesse einer ökonomischen, syntaktische Strukturen optimal ausnutzenden Rede klar wi-

2 Bezeichnend ist z. B. die folgende Aussage in Diewald/Steinhauer (2020: 58) zur Etablierung gendersprachlicher Innovationen im allgemeinen Sprachgebrauch: „Kurz, wir halten es für ein Gebot »revolutionärer List«, Veränderungen an dem Punkt zu beginnen, an dem am wenigsten Schwierigkeiten und der größte Erfolg zu erwarten sind." Aus der Beobachtung, welche Phänomene sich besonders leicht ausbreiten und wo die bestehende Sprachstruktur resistenter gegenüber Innovationen ist, lassen sich wertvolle typologische Rückschlüsse auf das Funktionieren von Sprachsystemen ziehen. 
dersprechen: Lexikalische Doppelungen wie Schülerinnen und Schüler sind unökonomisch, genderneutrale Umschreibungen z. B. mit Partizipien im Plural wie die Lernenden sind Beispiele eines schriftsprachlichen Nominalstils, bei dem Verben zunächst zu Nominalisierungen komprimiert und dann als Aktanten in Prädikationen eingesetzt werden. Diese komplexe Funktionalstilistik ist anfangs nur in engen Zirkeln von Aktivisten geformt worden. Wie es gelingt, breitere Sprecherkreise für ihre Anforderungen zu motivieren, stellt ein besonderes soziolinguistisches Erkenntnisinteresse dar. Dabei ist auch zu überlegen, wie nachhaltig und stabil dieser S p r a chwand el v on o be n sein kann. In diesem Zusammenhang spielen sprachpolitische Kriterien eine Rolle, die sich der linguistischen Interpretation entziehen. Während z. B. in Deutschland gendergerechtes Sprechen allmählich in vielen Bereichen des öffentlichen Lebens implementiert wird, gibt es in Frankreich schon seit mehreren Jahren den Ansatz, den Gebrauch gendergerechter Formen schrittweise aus dem öffentlichen Sprachgebrauch zu verbannen - und zwar explizit unter Verweis auf die oben erwähnte Komplexität der in diesem Stil präferierten sprachlichen Strukturen, die ganze Bevölkerungsgruppen von der Teilhabe am öffentlichen Leben ausschließen könnten (vgl. z. B. FAZ 07.05.2021). Der grammatischen Komplexität des gendergerechten Diskurses ist insofern als einem soziolinguistisch relevanten Phänomen vermehrt Aufmerksamkeit zu widmen.

Die vorliegende Abhandlung beschäftigt sich mit genderlinguistischen Aspekten in zwei konkreten Minderheitensprachen - dem Nieder- und Obersorbischen. Die sorbischen Sprachen sind in Abhandlungen zur Genderlinguistik bislang kaum vertreten. ${ }^{3}$ Für sie als Minderheitensprachen gelten besondere identitäts- und kulturpolitische Bedingungen: Der eigenen Sprache als identitätsstiftendem Faktor der Minderheitengesellschaft wird nicht nur innerhalb der sorbischen Gesellschaft selbst eine überragende Bedeutung beigemessen, obschon die gleiche Einstellung hinsichtlich der Mehrheitsgesellschaft als identitärer „Sprachkampf“ kritisiert würde (vgl. Lobin 2021: $125-128 ; 148) .{ }^{4}$ Dabei verfügt die sorbische Sprachgemeinschaft über ein

3 Einen Überblick zu genderlinguistischen Problemstellungen vor allem in der Flexionsmorphologie des Sorbischen sowie einen ersten Aufgabenkatalog für die sorabistische Genderlinguistik bietet Menzel (2021).

4 In diesem ,Messen mit zweierlei Maß““ ist das Erfordernis der ,,positiven Diskriminierung“ von Minderheiten bzw. Minderheitensprachen zu erkennen, die mit besonders 
hohes Normbewusstsein (Šatava 2005). Der Großteil der in den beiden sorbischen Sprachen veröffentlichten Drucktexte erscheint im Domowina-Verlag Bautzen, wo nach strikten Kriterien der schriftsprachlichen kodifizierten Norm lektoriert wird. Unter diesen relativ statischen Bedingungen ist die Aufnahme von gendergerechten sprachlichen Mitteln, sofern sie sich nicht genau an die kodifikatorischen Vorgaben halten, in die Schriftsprache nicht oder nur gegen erheblichen organisatorischen Widerstand möglich. Die Umgangssprache ist für Sprachwandel viel leichter zugänglich, aber sie ist bekanntlich den komplexen genderlinguistischen Ausdrucksstrategien nicht sehr affin (s. o.). Soziokulturelle Werte der sorbischen Gesellschaft erschweren die Ausbreitung nicht-binärer Genderkonzepte ebenfalls. Gerade auch mit Blick auf die Sprachvermittlung genießen Elternschaft und traditionelle Familien- und Sippenstrukturen in der sorbischen Gesellschaft einen hohen Stellenwert, soweit man das bei der kleinteilig gegliederten Lebenswelt der Sorben überhaupt pauschal sagen kann. ${ }^{5}$ Gegenüber feministischen Lebensentwürfen besteht oftmals eine kritische Distanz. Im Zuge von aktuellen Diversifizierungs- und Säkularisierungsprozessen ist diese aber nicht mehr unumstößlich. Es gibt sogar schon eine feministische sorbische Rap-Gruppe Kolektiw Klanki (siehe https://www.mdr.de/kultur/musik/kolektiw-klanki-sorbischer-rap-100.html [26.05.2021]). Wie sich zeigt, wächst auch in dieser zahlenmäßig kleinen und relativ geschlossenen Sprachgemeinschaft das Interesse an markierten genderdifferenzierenden bzw. genderneutralisierenden Ausdrucksmöglichkeiten in der eigenen Sprache.

starken kulturellen Rechten ausgestattet werden müssen, um ihre im Vergleich zur Mehrheitsgesellschaft üblicherweise schwachen ökonomischen Rahmenbedingungen ausgleichen zu können (Nekvapil 2007: 153 f.).

${ }_{5}$ Diese umfasst zumindest die traditionellen Untergruppen der katholischen Obersorben, der evangelischen Obersorben und der evangelischen Niedersorben (vgl. Menzel/ Pohontsch 2020). 


\section{Analysegegenstand und Korpusbeschreibung}

Das soziolinguistische Themenspektrum, das hier vorgestellt werden soll, betrifft genderlinguistisch relevante Strukturen des Sorbischen als Phänomene des Sprachkontakts. Es ist anzunehmen, dass die sprachlichen Mittel für die Realisierung des gendergerechten Sprechens vorzugsweise aus dem Deutschen übernommen werden, mit dem ja ein enger Sprach- und Kulturkontakt besteht. Allerdings unterscheiden sich die grammatischen Systeme dieser Kontaktsprachen wesentlich, nicht zuletzt hinsichtlich der Ausdrucksmittel für das Genus, das der sprachlichen Kodierung von Geschlechterkontrasten bei Substantiven mit der Denotation auf Personen und Tiere zugrunde liegt. Für sprachplanerische Handlungsempfehlungen wäre die Anlehnung an slavische Sprachen, vor allem an das Polnische sinnvoller, da die Genussysteme dieser genetisch verwandten Sprachen größere Übereinstimmungen aufweisen. Darauf wird in Menzel (2021: 120-125) ausdrücklich hingewiesen. In der betreffenden Arbeit werden die systemhaften Genuskontraste in den Flexionssystemen des Nieder- und Obersorbischen beschrieben, die eine genusindifferente bzw. genderneutrale Ausdrucksweise bei Personenbezeichnungen und Personennamen in vielen syntaktischen Kontexten unmöglich machen. Genderspezifische Wortformen kommen im Sorbischen (wie im Polnischen) aus flexionsmorphologischen Gründen viel häufiger vor als im Deutschen. Das gilt insbesondere für das Obersorbische, wo bei den Substantiven und den meisten kongruierenden Wortarten (mit Ausnahme der verbalen 1-Form) das maskulin-personale Subgenus im Plural unterschieden wird. Im Niedersorbischen sind die Genuskontraste im Plural großenteils abgebaut worden. ${ }^{6}$

Den Sprachvergleich mit Ausdrucksmitteln der gendergerechten Rede in anderen slavischen Sprachen kann auch die vorliegende Arbeit noch nicht leisten. Anhand einer kleinen Korpusanalyse inventarisiert sie genderlin-

${ }^{6}$ Zum Teil sind die entsprechenden Regelungen auch im Obersorbischen künstliche schriftsprachliche Normen. So besteht in der Deklination der Pronomen und Adjektive für den Nominativ Dual der Schriftsprache eine Unterscheidung nach personalen Maskulina und Non-Maskulin-Personalia (taj ,diese beiden [Männer]' bzw. tej, diese beiden [Frauen, Kinder, Tiere, Gegenstände]'), aber nur bei nicht-palatalen Stammauslauten. Die Verhältnisse in den Dialekten und in der Umgangssprache sind hingegen genderneutral, da sie die Personalitätskategorie im Nominativ Dual weitgehend aufgegeben haben (Fasske 1981: 691). 
guistisch relevante Strukturen, wie sie im Sprachgebrauch des Nieder- und Obersorbischen vorkommen. Dafür wird jeweils eine Ausgabe der niedersorbischen Wochenzeitung Nowy Casnik und der obersorbischen Abendzeitung Serbske Nowiny ausgewertet. Als Vergleichsgrundlage dienen die vierseitige Monatsbeilage der Serbske Nowiny in deutscher Sprache ${ }^{7}$ sowie einige Artikel in der ausgewerteten Nummer des Nowy Casnik auf Deutsch. Damit sind deutschsprachige Texte erfasst, die vom Umfang und Themenspektrum den beiden sorbischen Blättern ungefähr entsprechen und den gleichen Redaktionen entstammen wie die sorbischen Zeitungen. Im Einzelnen sind die folgenden Ausgaben berücksichtigt:

Tabelle 1. Das ausgewertete Textkorpus

\begin{tabular}{|l|l|c|c|c|c|}
\hline \multicolumn{1}{|c|}{ Sprache } & \multicolumn{1}{|c|}{ Name } & Nummer & Datum & Seiten & Wortformen \\
\hline niedersorbisch & $\begin{array}{l}\text { Nowy } \\
\text { Casnik }\end{array}$ & Jg. 72 Nr. 17 & 29.04 .2021 & S. 1, 2, 4-8 & 7934 \\
\hline obersorbisch & $\begin{array}{l}\text { Serbske } \\
\text { Nowiny }\end{array}$ & Jg. 31 Nr. 76 & 21.04 .2021 & S. 1-4 & 6277 \\
\hline deutsch & $\begin{array}{l}\text { Serbske } \\
\text { Nowiny }\end{array}$ & Jg. 31 Nr. 81 & 28.04 .2021 & S. 5-8 & 6985 \\
\hline deutsch & $\begin{array}{l}\text { Nowy } \\
\text { Casnik }\end{array}$ & Jg. 72 Nr. 17 & 29.04 .2021 & S. 2, 3, 7 & 1459 \\
\hline
\end{tabular}

In das Untersuchungskorpus gehen recht homogene publizistische Vergleichstexte ein. Das Korpus ist als Stichprobe des Sprachgebrauchs allerdings so klein, dass es keine quantifizierende Bewertung der untersuchten sprachlichen Formen gestattet. So werden im Folgenden allenfalls relative Auswertungen mit der gebotenen Vorsicht vorgestellt. Aufgrund seiner überschaubaren Größe ermöglicht dieses Korpus jedoch die Methode des "close reading". Im Verfahren der durchgängigen Textanalyse sollen sämtliche einschlägigen Textstellen mit Personenbezeichnungen ermittelt werden, um zu prüfen, welche genderlinguistisch relevanten Ausdrucksmittel in ihnen verwendet werden. Auf diese Weise können divergierende einzelsprachliche Präferenzen

${ }^{7}$ Hier handelt es sich um Paraphrasen und nicht oder nur in seltenen Fällen um direkte Übersetzungen von Zeitungstexten, die im Laufe des vorhergehenden Monats in obersorbischer Sprache erschienen sind. 
für bestimmte Optionen des gendergerechten Sprechens in den Vergleichssprachen, die sich aus den unterschiedlichen grammatischen Strukturen des Genusausdrucks ergeben, einander gegenübergestellt werden. Formulierungen nach dem Muster des Deutschen sind in diesem Zusammenhang ebenfalls leicht zu erkennen; bewerten lässt sich aber auch, ob sie im Sorbischen effizient sind. Außerdem lässt sich ermitteln, in welchen Fällen gendergerechtes Sprechen in diesen drei Stichproben $\mathrm{n}$ icht realisiert wird. Daraus kann man Rückschlüsse darauf ziehen, ob die Vorgaben der Genderlinguistik in unserem Untersuchungskorpus nur sporadisch oder doch mit einer gewissen Konsequenz berücksichtigt werden.

Genderrollen sind ausschließlich auf Menschen bezogen. Wenn wir Art und Ausmaß der Realisierungen von Ausdrucksmitteln des gendergerechten Sprechens analysieren wollen, müssen im Untersuchungskorpus also alle Arten von Personenbezeichnungen aufgesucht werden. Diese sind dann zu unterscheiden nach solchen Verwendungsweisen, in denen die Zugehörigkeit der gemeinten Person(en) zu einem binären Geschlecht (weiblich vs. männlich) durch die Genuskodierung Femininum vs. Maskulinum ausgedrückt wird, und nach Verwendungsweisen, in denen diese Unterscheidung fehlt. Eine interessante Vergleichsgröße ist die Vorkommenshäufigkeit von Personennamen im Text, bei denen zumindest auf der Grundlage der traditionellen geschlechtsspezifischen Namengebung in der Regel das Geschlecht des gemeinten menschlichen Individuums zu erkennen ist.

Eine Strategie zur Vermeidung geschlechtsspezifischer Ausdrucksweisen kann - ungeachtet ihres Wertes als Stilmittel - auch die Metonymie sein: wenn z. B. statt der handelnden Person die Institution genannt wird, der die Person zugeordnet ist. Verbreitet wird in genderlinguistischen Handreichungen gefordert, statt geschlechtsspezifischer Personenbezeichnungen geschlechtsindifferente Gruppenbezeichnungen bzw. Kollektiva zu verwenden (z. B. Diewald/Steinhauer 2020: 131; vgl. Kotthoff/Nübling 2018: 120). Das gilt ebenfalls für die Mittel der persönlichen Anrede (vgl. polnisch Droga społeczności ,Liebe Gesellschaft' im Vokativ), die in publizistischen Texten allerdings nicht häufig sind. In den vorliegenden Zeitungstexten sind innovative genusneutrale Anredeformen nicht enthalten.

Überhaupt ist festzuhalten, dass sich die gendersprachlichen Aktivitäten sowohl in den beiden sorbischen Sprachen als auch im Deutschen der sorbischen Zeitungsredaktionen strikt auf dem Boden der Sprachnorm bewe- 
gen und dass orthographische Markierungen der gendergerechten Sprache (*, / usw.) nur sehr behutsam verwendet werden. Auch genusneutralisierende Kodes in der Flexionsmorphologie (Lehrerx, Präsidentx) oder generische Feminina, wie sie in feministischen Texten auf Deutsch vorkommen, fehlen in unserem Korpus völlig.

Auf der Grundlage der vorgestellten Überlegungen werden in den ausgewerteten Zeitungstexten die folgenden Kontexttypen bzw. Fallgruppen inventarisiert:

1. Personennamen - in der Gliederung nach Frauen und Männern

2. Personenbezeichnungen mit der Referenz auf männliche oder weibliche Personen

3. Personenbezeichnungen ohne geschlechtsspezifische Referenz

4. Personenbezeichnungen ohne gegengeschlechtliches Äquivalent (englisch epicenes)

5. Bezeichnungen für Institutionen in metonymischer Funktion

In Gruppe 2 wird gesondert unterschieden nach Personenbezeichnungen, die im unmittelbaren Kontext eines Personennamens auftreten (z. B. Präsident Putin) und Personenbezeichnungen, bei denen das nicht der Fall ist (z. B. der Präsident Russlands). Der Geschlechterbezug ist in beiden Fällen gegeben, er wird durch die Nennung des Personennamens aber noch offensichtlicher. In den Fallgruppen 1 und 2 wurden Anforderungen des gendergerechten Sprechens im Sinne der Vermeidung konkreter Genus- bzw. Geschlechtszuweisungen durch sprachliche Formen offensichtlich nicht beachtet. Sie wären auch nur mit dem Mittel der Verschleierung der Geschlechtszugehörigkeit der gemeinten Person im Wege einer genusindifferenten Umschreibung mit einem Gruppennamen möglich gewesen (z. B. die russische Staatsführung).

Gruppe 3 bezeichnet das hauptsächliche Aufgabengebiet genderaktivistischer Sprachveränderung. Hier geht es um die Verwendung von Personenbezeichnungen ohne konkrete individuierende Referenz. In der Versprachlichung der betreffenden Proposition wird also ein Femininum oder (fast immer) ein Maskulinum gewählt, obwohl die Geschlechtszugehörigkeit der gemeinten Person(en) unbestimmt bleibt. Dies ist der Einsatzbereich des sogenannten generischen Maskulinums, das als unmarkiertes Substantiv eintritt, wenn die Geschlechtszugehörigkeit der personalen Referenten kommunikativ irrelevant ist. An diese Kontexttypen knüpfen sich die Forderungen nach der Sichtbarmachung von Frauen in der Sprache oder nach größerer 
Gendergerechtigkeit. Demgemäß ist dann, wenn beide Geschlechter der binären Genderkonzeption gemeint sein können, auch die Beidnennung von Maskulina und Feminina zu bevorzugen, oder wiederum die genderneutrale Umschreibung. Das Prinzip der Sprachökonomie wird also durch lexikalische Redundanz zugunsten der Eindeutigkeit von Geschlechtsreferenz zurückgedrängt. In die Gruppe 3 fallen aber auch Kontexte der morphologischen Neutralisierung von Genusunterschieden $-\mathrm{z}$. B. Pluralformen im Deutschen: die Deutsche $_{\text {fem. }}$ vs. der Deutsche mask. $_{\text {. }}$ Sg.) $=>$ die Deutschen mask./fem. $_{\text {Dl.). Hier }}$ zeigen sich deutliche, durch strukturelle Kontraste der einzelsprachlichen Flexionssysteme bedingte Unterschiede zwischen den drei Vergleichssprachen. Diese Kontexte werden im Folgenden eigens vorgestellt und ausgezählt.

In der vierten Gruppe finden sich unterschiedliche Klassen von Substantiven, die keine paarigen Geschlechtergegensätze ausdrücken. Hier sind zahlreiche Lexeme vertreten, die von Genderaktivisten als Ersatzstrategie für die genusspezifischen Personenbezeichnungen vorgeschlagen werden. Sie können allen drei Genera angehören; z. B. im Deutschen Maskulina der Mensch, der Vorstand, Feminina die Lehrkraft, die Familie oder Neutra das Mitglied, das Publikum. Auch Pluraliatantum sind erfasst (z. B. die Eltern, (junge) Leute, die Fachleute als suppletiver Plural zu der Fachmann und (seltener) die Fachfrau). In bislang nur seltenen Fällen ist in dieser Gruppe diachroner Wandel zu beobachten, wenn z. B. zum deutschen geschlechtsneutralen Substantiv der Gast (mask.) ein innovatives Femininum die Gästin gebildet wird, so dass diese beiden Substantive in die Gruppe 2 (Personenbezeichnungen mit geschlechtsspezifischer Referenz) übertreten. ${ }^{8}$ Ob ein Substantiv ein gegengeschlechtliches Äquivalent bildet oder nicht, muss anhand von Wörterbüchern geprüft werden. Für die beiden sorbischen Sprachen werden diesbezüglich die einschlägigen Online-Wörterbücher in beiden Richtungen (nieder-/obersorbisch-deutsch, deutsch-nieder-/obersorbisch) konsultiert: ${ }^{9}$ Z. B. gibt es zu os. čłónk ,Mitglied“ ein Feminati-

8 Analog hat die Rada Języka Polskiego unlängst den Neologismus gosćini ,Gästin“ für die polnische Schriftsprache zugelassen, welcher offenbar durch das Einwirken einer einzelnen Sprachaktivistin, Kazimiera Szczuka, in den allgemeinpolnischen Genderdiskurs eingebracht wurde (Wąsowska 2020). Zur Wortgeschichte von Gästin s. auch Menzel (2021: 122).

9 Vgl. niedersorbisch.de (in beide Richtungen), soblex.de (obersorbisch-deutsch), www.hornjoserbsce.de/dow/ (deutsch-obersorbisch) [26.05.2021]. 
vum čłonča (Fallgruppe 2), zu ns. cłonk ,dss.' jedoch nicht (Fallgruppe 4). - Substantive, die zwar kein mit morphologischen Mitteln gebildetes gegengeschlechtliches Äquivalent haben, deren Geschlechtsreferenz aber semantisch konstitutiv ist (z. B. der Mann/Vater, die Frau/Mutter) werden hier nicht berücksichtigt - wohl aber das Kind als genderneutrales Wort (da es männliche und weibliche Kinder gibt).

Die fünfte Fallgruppe enthält einige metonymische Kontexte, die der genderlinguistischen Strategie der umschreibenden Verschleierung von Geschlechtsidentitäten entsprechen. Hier können allerdings nur relativ konkrete Umschreibungen aufgenommen werden, die Elemente der Handlungsstruktur aus der Proposition mit der explizit versprachlichten Personenbezeichnung beibehalten. So werden nur Sätze mit Handlungsverben berücksichtigt, vor allem mit verba dicendi (z. B. die Regierung ließ mitteilen statt die Regierungssprecherin / der Regierungssprecher teilte mit). In diesen Konstruktionen ist die Substitution einer geschlechtsspezifischen Personenbezeichnung relativ offenkundig. Genderlinguistisch präferente Umschreibungen greifen häufig auch auf Abstrakta zurück, wobei der personale Charakter der Äußerung verloren gehen kann (das Gesundheitsamt registrierte 136 Neuinfektionen statt Mitarbeiter / Mitarbeiterinnen des Gesundheitsamts registrierten 136 infizierte Personen). Kontexte, in denen keine Bezeichnungen von Personen und Organisationen vorkommen, werden nicht in die Untersuchung einbezogen, zumal wenn sie ohne verbum dicendi stehen.

Schließlich werden in einer sechsten Gruppe die im Korpus enthaltenen Kontexte der genderlinguistisch präferenten Beidnennung gezählt, die als Einzellexeme in den Gruppen 2 oder 3 enthalten sind.

6. Beidnennung von Geschlechterkontrasten

Diese Fälle verdienen besondere Beachtung, da sie auf explizite Weise dokumentieren, dass die Urheber der ausgewerteten Texte den Ansprüchen der gendergerechten Sprache nachkommen. Wer diese lexikalisch redundante Ausdrucksweise pflegt, unterstreicht damit seine besondere Verbundenheit mit den Zielsetzungen von sprachlichen Genderaktivisten. Der Einzelanalyse sei vorausgeschickt, dass solche Strukturen in allen drei Vergleichssprachen des Korpus überraschend selten sind und nur bei einzelnen Autoren auftreten. 


\section{Materialauswertung}

In den drei Teilkorpora wurden die folgenden Belegzahlen ermittelt. Angegeben sind die Werte pro 1000 Wortformen im Einzelkorpus und in Klammern die absoluten Zahlen.

Tabelle 2. Korpusauswertung der für gendergerechte Sprache relevanten Kontexte

\begin{tabular}{|c|c|c|c|c|c|c|}
\hline \multirow{2}{*}{} & \multicolumn{2}{|c|}{ niedersorbisch } & \multicolumn{2}{c|}{ obersorbisch } & \multicolumn{2}{c|}{ deutsch } \\
\cline { 2 - 7 } & fem. & mask. & fem. & mask. & fem. & mask. \\
\hline $\begin{array}{c}\text { 1) Personennamen } \\
\text { (PN) }\end{array}$ & $\begin{array}{c}14,4 \\
(114)\end{array}$ & $11,0(87)$ & $4,5(28)$ & $13,1(82)$ & $7,7(65)$ & $14,1(119)$ \\
\hline 2) Pers.Bez. +ref. & $9,1(72)$ & $5,4(43)$ & $4,6(29)$ & $10,7(67)$ & $5,9(50)$ & $11,3(95)$ \\
\hline davon mit PN & $1,8(14)$ & $2,3(18)$ & $1,8(11)$ & $4,9(31)$ & $2,3(19)$ & $7,7(65)$ \\
\hline 3) Pers.Bez. -ref. & - & $10,1(80)$ & $0,2(1)$ & $17,8(112)$ & - & $8,2(69)$ \\
\hline genusindifferent & \multicolumn{2}{|c|}{$0,9(7)$} & $2,5(16)$ & $2,4(20)$ \\
\hline 4) epicenes & \multicolumn{2}{|c|}{$10,0(79)$} & \multicolumn{2}{c|}{$6,9(43)$} & $7,0(59)$ \\
\hline 5) Metonymien & \multicolumn{2}{|c|}{$1,3(10)$} & \multicolumn{2}{c|}{$2,2(14)$} & $2,5(21)$ \\
\hline 6) Beidnennungen & \multicolumn{2}{|c|}{$0,4(3)$} & \multicolumn{2}{c|}{$44,3(8)$} & \multicolumn{2}{c|}{$0,2(2)$} \\
\hline n (ohne PN) & \multicolumn{2}{|c|}{$36,7(291)$} & \multicolumn{3}{c|}{$37,2(314)$} \\
\hline
\end{tabular}

Die Vergleichsgruppe der Personennamen weist beim Verhältnis von männlichen und weiblichen Personen große Schwankungen in den Teilkorpora auf. In den obersorbischen Texten kommen fast dreimal so viele Männer- wie Frauennamen vor, in den deutschen sind erstere fast doppelt so häufig wie letztere, nur in den niedersorbischen Texten dominieren die Frauennamen. Diese Unregelmäßigkeiten können durch das Themenspektrum zu erklären sein, das in den Zeitungen behandelt wird. ${ }^{10}$ Sie zeigen aber auch, dass das vorgelegte Korpus für quantitative Auswertungen noch viel zu klein ist.

10 Die Belange außerhalb der Lausitz und weltpolitische Ereignisse mit überwiegend männlichen Akteuren werden nur in den obersorbischen Texten der Serbske Nowiny beschrieben. Bei der Durchsicht des niedersorbischen Nowy Casnik fällt auf, dass der Schwerpunkt der Darstellung eindeutig auf lokalen Verhältnissen in den niedersorbischen Dörfern liegt, in denen zahlreiche weibliche Akteure zu nennen sind. 
Die Zahl der ausgewerteten appellativischen Personenbezeichnungen (Gruppe 2-5 zusammen) schwankt nur um den Faktor 1,2 - im obersorbischen Korpus treten diese Kontexte ungefähr um $20 \%$ häufiger auf als im niedersorbischen und deutschen Korpus.

Die Anzahl der Personenbezeichnungen, die geschlechtsspezifisch mit Referenz auf Frauen oder Männer verwendet werden (Fallgruppe 2), ist durchweg kleiner als die der Personennamen (mit einer Ausnahme bei den Frauenbezeichnungen im Obersorbischen, wo weibliche Namen und Appellativa gleichermaßen selten sind). Interessant ist, dass ein beträchtlicher Teil dieser Appellativa mit Personennamen steht, wenn sie sich auf Männer beziehen (ca. 40-70 \%), bei Frauen ist dieser Anteil geringer (ca. 20-40 \%). Auf Männer wird also häufiger als auf Frauen in Zusammenhang mit ihrem Beruf oder ihrer gesellschaftlichen Funktion referiert. Dagegen wird die Untergruppe der weiblichen Personenbezeichnungen häufig durch Verwandtschaftsbezeichnungen oder Charakterisierungen bereits in den Diskurs eingeführter Personen ausgefüllt (s. die Beispiele 1-2 mit Frauenbezeichnungen ohne Eigennamen). Movierte Feminative als Berufsbezeichnungen sind relativ selten; vgl. Beispiel (3) in der Unterschrift zur Anzeige einer Kultureinrichtung, die zuerst auf Deutsch und dann auf Niedersorbisch abgedruckt wird, so dass der Einfluss des Deutschen auf die niedersorbische Formulierung offensichtlich ist. ${ }^{11}$ Besonders selten werden Feminative im Plural verwendet (Beispiel 4 Osterreiterinnen, wo die Markiertheit des Feminativums im Kontrast zur zweimaligen Verwendung eines generischen Maskulinums besonders offenkundig wird).

(1) ns. We tej kšušcynje w slěpjańskich stronach se zmakaju chódoty. (NC 72/17: 6) ,In diesem Birnbaum in der Schleifer Gegend treffen sich die Hexen.

(2) os. „Naša mać bě klankodźiwadźelnica“, 44lětna rozprawja, [...] (SN 31/76: 3) „,Unsere Mutter war Puppentheaterschauspielerin“, erläutert die 44-Jährige, $[\ldots]^{6}$

11 Die Anrede in der gleichen Zeitungsannonce wird weder in der deutschen noch in der niedersorbischen Version gendergerecht formuliert (Fallgruppe 3): Liebe Freunde der sorbischen Sprachschule, [...] / Lube pśijaśele serbskeje rěcneje šule, [...]. 
(3) dt. Die Mitarbeiterinnen der Schule für Niedersorbische Sprache und Kultur / ns. Sobuźétaśérki Šule za dolnoserbsku rěc a kulturu (NC 72/17: 7)

(4) dt. Unter den wiederkehrenden Fragen, die die Teilnehmer den Referenten stellten, lösten mögliche Osterreiterinnen die größte Resonanz aus. (SN 31/81: 8)

Der größte Teil dieser Personenbezeichnungen mit individuierter Referenz steht jedenfalls im Singular. Bei den Pluralformen ist die genderspezifische Gebrauchsweise oft nur aus dem Kontext zu ersehen, z. B. wenn im Sportteil einer Zeitung über einen Fußballverein mit den Substantiven Delnołužičanojo ,Niederlausitzer', hrajerjo ,Spieler', koparjo „Fußballspieler' und Łužičenjo ,Lausitzer' berichtet wird. Da man weiß, dass es sich um einen Männerfußballverein handelt, ist zu schließen, dass diese Personenbezeichnungen konkret Männer bezeichnen und nicht im generischen Maskulinum gemeint sind. - Aufschlussreich sind in diesem Zusammenhang auch die folgenden Textstellen im Numerus Dual:

(5) ns. Historikarja Friedrich Pollack (pšawy) a Jana Piňosová wót Serbskego instituta we wustajeńcy w Serbskem muzeju. (NC 72/17: 1) ,Die beiden Historiker Friedrich Pollack (rechts) und Jana Piňosová vom Sorbischen Institut in der Ausstellung im Sorbischen Museum. os. Połdra lěta staj historikarjej SI dr. Jana Piňosová a dr. Friedrich Pollack [...] na taflowej prehladce dźéłałoj. (SN 31/76: 1), Eineinhalb Jahre arbeiteten die beiden Historiker des SI Dr. Jana Piňosová und Dr. Friedrich Pollack [...] an der Bildausstellung.“

Die maskuline Berufsbezeichnung historikar ,Historiker' steht beide Male im Dual Maskulinum, sie wird jeweils durch einen männlichen und einen weiblichen Personennamen (in unterschiedlicher Abfolge) erweitert. Das entspricht völlig den Genuszuweisungsregeln (gender resolution rules; Corbett 1991: 261-306) im Sorbischen, welche besagen, dass sowohl im Plural als auch im Dual die maskuline (niedersorbisch) bzw. maskulin-personale (obersorbisch) Form zu wählen ist, wenn auch nur einer der Referenten männlich ist (vgl. Fasske 1981: 398). Der Gebrauch der seltenen flexivischen Dualformen ist beim Substantiv präferent. Bei einer dualischen Konzipierung 
der Numerusverhältnisse lässt sich allerdings der Geschlechtsunterschied der genannten Personen nicht mit grammatischen Mitteln ausdrücken. Würde die gendergerechte Beidnennung eingesetzt, müssten die Substantive im Singular stehen, und der flexivische Dual wäre auf die Verbform beschränkt (os. staj historikar F. P. a historikarka J. P. dźéłałoj). - Im niedersorbischen Text findet sich noch ein zweites, analoges Beispiel für den Dualgebrauch im Genderkonflikt:

(7) ns. Českej stipendiata na Instituśe za sorabistiku Martin Boukal a Anna Kopová stej se teke pśizjawitej z krotkim pśinoskom [...] (NC 72/17: 4) ,Die beiden tschechischen Stipendiaten am Institut für Sorabistik Martin Boukal und Anna Kopová meldeten sich ebenfalls mit einem kurzen Beitrag [...]

Ohne geschlechtsspezifische Referenz gebrauchte Personenbezeichnungen (Fallgruppe 3) sind in den meisten Fällen pluralisch. Die wenigen Ausnahmen im Singular sind oft syntagmatisch motiviert (os. kóždy sportowc,jeder Sportler ${ }^{6}$ ); aber auch singularische Klassenreferenz ist möglich wie im folgenden Beispiel:

(8) ns. Familija nadawkdawarja wiżi tapetu lěta dtujko na sćěnje (NC 72/17: 8) ,Die Familie eines Auftraggebers sieht die Tapete jahrelang an der Wand'

Bei den hier zusammengestellten Formen geht es nicht um pragmatische Definitheit und Referenzialität, sondern um die Bewertung der Frage, ob aus der Nennung der Wortform im konkreten Kontext ein Rückschluss auf das Geschlecht des Referenten möglich ist oder nicht. In Beispiel (8) ist das nicht der Fall, denn die Aussage kann sich auch auf weibliche Auftraggeber beziehen. Aufgrund dieser Fragestellung treten Feminina in Fallgruppe 3 praktisch nicht auf. Das Femininum ist schließlich das funktional markierte Genus, das bei Personenbezeichnungen in der Regel positiv die weiblichen Wesen bezeichnet und nicht geschlechtsneutral eingesetzt wird. Ein singuläres Gegenbeispiel ist das Folgende: 
(9) os. prezident konferency wysokošulskich rektorow, zastupnicy zajimow uniwersitow w Němskej, Peter-André Alt (SN 31/76: 2) ,der Präsident der Hochschulrektorenkonferenz, der Vertreterin der Interessen der Universitäten in Deutschland, Peter-André Alt ${ }^{\star}$

Hier liegt prinzipiell die in Fallgrupe $5 \mathrm{zu}$ behandelnde metonymische Verwendung eines Organisationsnamens vor, dessen Grundwort konferenca ,Konferenz" ein Femininum ist. Es wird in einer Apposition mit dem gegengeschlechtlichen Wortpaar zastupnik/zastupnica ,Vertreter/Vertreterin“ durch das Femininum aufgenommen, um die semantischen Beziehungen in dieser komplexen syntaktischen Struktur durch die Genusstabilität der anaphorischen Bezüge zu verdeutlichen: schließlich geht es um die Tätigkeitscharakterisierung der Hochschulrektorenkonferenz (Femininum), nicht ihres Präsidenten (Maskulinum).

Ansonsten enthält Fallgruppe 3 überwiegend Verwendungen generischer Maskulina - also substantivischer Personenbezeichnungen des Genus Maskulinum, die sich auf männliche oder weibliche Personen beziehen können. Sie machen in allen drei Korpora einen bedeutenden Teil aller hier zusammengestellten Personenbezeichnungen aus $(22,0 \%$ im deutschen, 27,5 \% im niedersorbischen und sogar 39,7 \% im obersorbischen Teilkorpus), was verdeutlicht, dass gendergerechte Beidnennungen in den untersuchten Texten nicht zum stilistischen Standardrepertoire gehören. Zwei weitere Belege:

(10) ns. Jadnab 20 demonstrantow jo protestěrowało (NC 72/17: 2) ,Etwa zwanzig Demonstranten protestierten

(11) os. Hdźež bě něhdy skała a pozdźišo črjopjenc, su préceljo hwězdarnje kerki wotstronili. (SN 31/76: 4) ,Wo früher ein Steinbruch und dann ein Schuttabladeplatz war, haben die Freunde der Sternwarte Büsche beseitigt.‘

An Stellen wie diesen wäre es ohne weiteres möglich, Beidnennungen einzusetzen (ns. demonstrantow a demonstrantkow, os. prećeljo a prećelki hwězdarnje). Ob sie zufällig fehlen oder bewusst vermieden werden, ist nicht zu entscheiden. Im deutschsprachigen Teilkorpus sind diese generischen Maskulina jedenfalls etwas seltener als im niedersorbischen und deutlich seltener als im obersorbischen Teilkorpus. Ihre geringe Zahl könnte eine stilistische 
Präferenz zugunsten der gendergerechten Sprache im Deutschen aufzeigen, da letztere in der deutschen Publizistik allgemein schon recht verbreitet ist.

Der Fallgruppe 3 sind ebenfalls die aus flexionsmorphologischen Gründen genusindifferenten Wortformen der Lexeme mit der Semantik von Personenbezeichnungen zugeordnet. Im Deutschen sind in diese Rubrik fast ausnahmslos die zahlreichen substantivierten Adjektive und Partizipien einzureihen, die im Plural keine Genusunterschiede aufweisen: die 16-Jährigen, Delegierte, Deutsche, Dumme, Ehrenamtliche, Interessierte, Jugendliche, die Samen, die Teilnehmenden, die Verantwortlichen. Obwohl das Niedersorbische in der Pluralflexion ebenfalls keine (oder nur marginale; s. Janaš 1984: 73) Genusunterscheidungen vornimmt, sind die analogen Fälle im niedersorbischen Teilkorpus selten vertreten: $z a$ dorosćonych ,für Erwachsene', ewangelske ,Evangelische', mlodostne ,Jugendliche', pótrjebnym ${ }_{\text {Dat. }}$ Pl. , den Bedürftigen', pśibytne ,die Anwesenden'. Alle fünf Lexeme können Lehnübersetzungen aus dem Deutschen sein. In der obersorbischen Zeitungsausgabe ist eine viel stärkere Präferenz für publizistisch-administrative Sprachformen festzustellen. Deshalb sind die substantivierten Adjektive und Partizipien auch recht häufig vertreten. Ein großer Teil davon ist aber genussensitiv - nämlich die maskulin-personalen Wortformen im Nominativ Plural, die mit elf Lexemen belegt sind: dalši z wěstymi předschorjenjemi, Weitere mit bestimmten Vorerkrankungen', domoródni ,Einheimische', druzy ,Andere', njeznaći ,Unbekannte', potrjecheni ,Betroffene', pristajeni ,Angestellte', přićehnjeni ,Zugezogene', sobuwojowacy ,Mitstreitende', zamotwići ,Verantwortliche', zwonkastejacy ,Außenstehende', zwólniwi ,die Bereitwilligen: Genusindifferent sind nur die Wortformen des Genitivs, Dativs, Instrumentals und Lokativs Plural (s. Menzel 2021), die in weiteren zwölf Belegen mit zehn Lexemen im obersorbischen Korpus verzeichnet werden: mlodostnych $_{\text {Gen.P. }}$, der Jugendlichen', natyknjenych $h_{\text {Gen.Pl. }}$, der Angesteckten', nimo ducym ${ }_{\text {Dat.Pl. }}$,den Vorbeigehenden', potrjechenych ${ }_{\text {Gen.PI. }}$, der Betroffenen', schorjenych ${ }_{\text {Gen.PI. }}$, der Erkrankten', stabym ${ }_{\text {Dat.PI. }}$, den Schwachen', studowacych $_{\text {Gen.Pl. }}$, der Studierenden', šćépjenych ${ }_{\text {Gen.PI. }},-y m_{\text {Dat.Pl. }}$, der, den Geimpften', testowanym $m_{\text {Dat.PI. }}$, den Getesteten', zapućowanych ${ }_{\text {Gen.Pl. }}$, der Zuwanderer. Auch in allen diesen Fällen bestehen lexikalische Parallelen mit dem Deutschen. Schließlich finden sich als flexionsmorphologische Besonderheit auch noch die Wortformen přishušnicy ,Angehörige', zastojnicy ,Beamte ${ }^{‘}$ und zastupnicy, Vertreter', die formal genusindifferent den Nominativ Plural von 
přistušnik ,Angehöriger` / přistušnica ,Angehörige', zastojnik ,Beamter / zastojnica ,Beamtin“ bzw. zastupnik ,Vertreter' / zastupnica ,Vertreterin“ ausdrücken können. Ungeachtet der Tatsache, dass die genusindifferenten Flexionsformen im Obersorbischen ganz anders verteilt sind als im Deutschen, kann das Obersorbische nach dem lexikalischen Vorbild des Deutschen doch eine überraschend große Zahl an genderneutralen Korpusbelegen aufbieten. Dabei behalten die Zeitungsredaktionen ihre unbedingte Orientierung an der geltenden Sprachnorm bei und geben nicht zu erkennen, dass künstliche genderneutrale Innovationen (s. o.), wie sie auch für das Polnische bisweilen vorgeschlagen werden, ${ }^{12}$ im Textgebrauch Einzug halten könnten.

Fallgruppe 4 enthält Personenbezeichnungen, bei denen ein gegengeschlechtliches Äquivalent im Wortschatz fehlt. Obwohl sie formal einem Genus zugeordnet werden, können keine am Geschlechterkontrast orientierten lexikalischen Paare gebildet werden. In diesem Sinne sind sie in den Bestand gendergerechter sprachlicher Ausdrucksmittel einzubeziehen. Das lexikalische Inventar dieser Rubrik wurde oben (s. 2.) mit Blick auf das Deutsche erläutert. Im Niedersorbischen enthält es u. a. eine Reihe von Maskulina: cłonk ,Mitglied', gósć ,Gast', kupc ,Käufer, Kaufmann', lud ,Volk', zajmc ,Interessierte(r)', znank,Zeuge/Zeugin'. Im Obersorbischen unterscheidet sich der lexikalische Bestand dieser Gruppe teilweise vom Niedersorbischen, da im Obersorbischen einige Substantive genus- und geschlechtsbasierte Paare bilden, wie sie im Niedersorbischen nicht sprachgerecht sind (s. o.: os. čłonka ,weibliches Mitglied', aber auch kupcowka ,Käuferin'). Immerhin verbleiben die folgenden acht nicht-paarbildenden Lexeme im obersorbischen Belegkorpus: čłowjek ,Mensch', dorost „Nachwuchs', hósć ,Gast', lud ,Volk', paduch ,Dieb', pěšk ,Fußgänger', sobustaw ,Mitglied', team ,Team‘. Auch in diesem Bereich sind lexikalische Einflüsse aus dem Deutschen anzunehmen, wie schon die gute Übersetzbarkeit dieser Lexeme nahelegt. Aufgrund deutlicher lexikalischer Idiosynkrasien in den Vergleichssprachen erübrigt sich die detailliertere Auswertung.

Die ebenfalls oben unter (2.) bereits erläuterten Metonymien sind seltene und bisweilen auch amtssprachliche Wendungen. Sie sollen hier nicht weiter systematisiert werden. Exemplarisch seien zwei Beispiele vorgestellt:

12 Zum Beispiel auf der Internetseite https://zaimki.pl/ [abgerufen am 21.05.2021] mit einem imposanten Katalog genusindifferenter Paradigmen von Pronominalendungen. 
(12) ns. Pó namšy wósada a faraŕka pśepšosyjotej do farskego gumna. (NC 72/17: 7) ,Nach dem Gottesdienst laden die Gemeinde und die Pfarrerin in den Pfarrgarten ein.

(13) os. [...] Moskwa informowaše knježerstwo Čěskeje, zo dyrbi so 20 zastojnikow jeje wulkopóstanstwa do Prahi wróćić. (SN 31/76: 2) ,Moskau informierte die Regierung Tschechiens, dass 20 Beamte ihrer Botschaft nach Prag zurückkehren müssten.

Das niedersorbische Beispiel (12) ist dadurch aufschlussreich, dass das Substantiv wósada ,Gemeinde', die Bezeichnung einer organisatorischen Einheit bzw. Gruppe, mit der Personenbezeichnung fararka ,Pfarrerin' koordinativ gebraucht werden kann. Die funktionale Äquivalenz unterstreicht hier die inhaltliche Vergleichbarkeit. Beispiel (13) aus dem Obersorbischen ist ein typischer Kontext mit einem verbum dicendi, bei dem der metonymische Charakter der Bezeichnung einer Organisationsstruktur (hier Moskau als Übertragung auf den Ort, an dem die Organisation angesiedelt ist) als agentiver Partizipiant (Moskau spricht) besonders klar zum Ausdruck kommt.

Wie oben bereits erläutert, sind die tatsächlich belegten Fälle von Beidnennung in die obige Auszählung bereits aufgenommen worden; hier werden sie (als Fallgruppe 6) nur aufgrund ihrer Bedeutung für die Ermittlung gendergerechter Ausdrucksmittel nochmals zusammengestellt. Es handelt sich allerdings um die am schwächsten vertretene Rubrik in der Übersicht von Tabelle (2). In den ausgewerteten deutschsprachigen Texten benutzt nur eine einzelne Autorin dieses stilistische Mittel, es ist ein nahezu idiolektales Element. So finden sich hier auch nur zwei Belege: Sorbinnen und Sorben in einem offensichtlich aus dem amtssprachlichen Originaltext übernommenen Zitat und die einzige Schreibung mit Binnen-I im gesamten Untersuchungskorpus: RednerInnen.

Die niedersorbischen Belege sind wobydlarje a wobydlarki bzw. wobydlarje a wobydlarki ,Einwohner und Einwohnerinnen' (mit orthographischen Varianten) sowie źowća a gólcy, Mädchen und Jungen'. Im Obersorbischen finden sich zwei graphische Besonderheiten: erstens sam/a ,allein mask./fem. ' in Joggować je dowolene, ale jeno sam/a ,Joggen ist erlaubt, aber nur allein' sowie die weitere Bindestrichschreibung moje kolegowki / moji kolegojo ,meine Kolleginnen / meine Kollegen' in einem Interview, das aus dem Deutschen übersetzt wurde. Die anderen sechs Beispiele sind inhaltlich und formal im 
Rahmen des Üblichen. Mindestens drei dieser Beispiele stammen ebenfalls aus Texten, die in der deutschen Übersetzungsvorlage schon die gendergerechte Beidnennung aufweisen könnten. Wie instabil diese Strukturen sind, zeigen die Schwankungen bei der Voranstellung der weiblichen oder männlichen Personenbezeichnungen. Vgl.: $k$ dalšim sportowčam a sportowcam ,zu weiteren Sportlerinnen und Sportlern', mjez sportowčemi a sportowca$m i$,zwischen den Sportlerinnen und Sportlern', šulerki a šulerjo ,Schülerinnen und Schüler', šulerjo a šulerki ,Schüler und Schülerinnen', wučerki a wučerjo ,Lehrerinnen und Lehrer' sowie das referenzielle wědomostnica a wědomostnik, die Wissenschaftlerin und der Wissenschaftler' als einzige Singularform in dieser Fallgruppe. Die Anzahl der einschlägigen Formen ist mit acht Belegen nicht so gering, dass diese übersehen werden könnten (was in Bezug auf das Niedersorbische durchaus möglich wäre). Ihre uneinheitliche formale Ausgestaltung deutet aber darauf hin, dass es sich mehr um emblematisches Symbolhandeln als um etablierte Redestrukturen handelt.

\section{Bewertung}

In dieser kleinen Korpusstudie wurde eine Reihe von Merkmalen gendergerechter Sprache im Sinne von Ausdrucksmitteln untersucht, die den Geschlechterunterschied männlich vs. weiblich bei Personenbezeichnungen kodieren. Zu unterscheiden ist dabei zwischen expliziten und impliziten Ausdrucksmitteln. Zu den expliziten gehört die Beidnennung wie Schülerinnen und Schüler oder die (bewusste) Verwendung von Abstrakta, welche die Geschlechterunterscheidung verschleiern (die Schülerschaft). Diese sind in den ausgewerteten Texten erstaunlich selten vertreten. Die geltenden orthographischen und grammatischen Regeln des Nieder- und Obersorbischen werden auch im Zusammenhang der gendergerechten Sprache prinzipiell beachtet. Implizite Ausdrucksmittel sind die Verwendung von Personenbezeichnungen ohne derivativisch zu kodierenden paarigen Geschlechterkontrast (z. B. als Feminina: ns./os. delegacija ,Delegation', ns./os. policija ,Polizei') und von paradigmatischen Wortformen, deren Endungen genusübergreifend gebraucht werden (ns. pśibytne, die Anwesenden', os. studowacych, der Studierenden'). Auch diese sind nicht häufig: im obersorbischen Korpus sind sie etwas besser vertreten als im niedersorbischen, obwohl die Anzahl der genusneutralen Positionen im Flexionssystem des Obersorbischen geringer ist 
als im Niedersorbischen. Das hat offensichtlich stilistische Gründe: Die vorliegende Ausgabe des niedersorbischen Nowy Casnik enthält eine Reihe feuilletonistischer Artikel, in denen unökonomische und abstrakte Ausdrucksweisen unangemessen wären. Allerdings weist die ausgewertete niedersorbische Publizistik sogar in Texten zu administrativen Themen weniger gendergerechte Formulierungen auf als die obersorbische.

Alle anderen Strategien des Ausdrucks von Genus und Geschlecht sind gegenüber den Anforderungen der Genderlinguistik neutral oder sogar ihnen entgegengerichtet. Überraschend häufig ist das generische Maskulinum zu verzeichnen, das meistens in pluralischen Kontexten, bisweilen aber auch im Singular gesetzt wird. Immerhin lassen sich keine Fälle beobachten, in denen auf eine individuierte weibliche Person mit der männlichen Variante einer Positions- oder Berufsbezeichnung referiert würde. Es heißt immer ns. šoltowka ,Bürgermeisterin', mólaŕka ,Malerin', mólaŕska mejstaŕka ,Malermeisterin' bzw. os. ministerka ,Ministerin', awtorka ,Autorin' oder architekt$k a$,Architektin', mit oder ohne Personennamen. Im Nowy Casnik wird sogar das auch im Deutschen bekannte Wort djane mit der Aussprache „didźejn“ eingeführt als feminative Entsprechung zum Anglizismus DJ [Discjockey].

Wie die angeführten deutschen Übersetzungsäquivalente belegen, sind die genderlinguistisch einschlägigen Formen in den beiden sorbischen Teilkorpora stark durch das Deutsche beeinflusst. Das ist auch ein Grund für ihren unregelmäßigen Gebrauch. Oben wurde an mehreren Stellen darauf hingewiesen, dass die Verwendung der explizit gendergerechten Formen an die stilistischen Präferenzen einzelner Autoren gebunden ist oder in Übernahmen (Übertragungen, Übersetzungen) aus dem Deutschen besonders oft vorkommt. Sorbische Originaltexte enthalten dem ersten Eindruck nach diese Merkmale seltener, was anhand einer nach Textgattungen differenzierten Studie zu prüfen wäre. Selbst im deutschsprachigen Teilkorpus gibt es große Unterschiede bei der Beachtung der gendergerechten Sprache. Hier finden sich einige Texte, in denen nach deutschem Standard gegendert wird; in der Mehrzahl der Texte fällt allerdings eher das Fehlen jeglicher Ausdrucksmittel der gendergerechten Sprache auf.

Stilistisch gereicht die Verwendung von Ausdrucksmitteln der gendergerechten Sprache den betreffenden Texten nicht zum Vorteil. Zumindest wird der Bezug auf das sprachliche Vorbild des Deutschen deutlich unterstrichen: Gendergerechte Sprache im Sorbischen ist ein deutlich erkennbares Phä- 
nomen des Sprach- und Kulturkontakts, dem die Originalität autochthoner sprachlicher Mittel fehlt. Außerdem erweist sich, dass diese Wendungen in Artikeln auftreten, deren Inhalt für amtssprachliche Formulierungen besonders zugänglich ist (Berichte aus Behörden und Institutionen, Stipendienausschreibungen $u$. ä.). In breiteren informellen Kreisen sind die sprachlichen Forderungen der genderpolitischen Aktivisten bei den Sprechern des Sorbischen offensichtlich nicht angekommen - und auch nur sehr bedingt in ihren deutschsprachigen Texten.

\section{Literatur}

Anttila R., 1989, Historical and comparative linguistics. 2nd edition, Amsterdam/ Philadelphia: Benjamins.

Corbett G. G., 1991, Gender, Cambridge (UK): University Press.

Diewald G., Steinhauer A., 2020, Handbuch geschlechtergerechte Sprache. Wie Sie angemessen und verständlich gendern, Berlin: Dudenverlag.

FASSKE H., 1981, Grammatik der obersorbischen Schriftsprache der Gegenwart. Morphologie, Bautzen: Domowina-Verlag.

FAZ 07.05.2021: Frankreich verbietet schriftliches Gendern an Schulen. In: Frankfurter Allgemeine Zeitung, [online:] https://www.faz.net/aktuell/politik/ausland/frankreich-verbietet-schriftliches-gendern-an-schulen-17332003.html, [02.08.2021]

JANAŠ P., 1984, Niedersorbische Grammatik für den Schulgebrauch, Bautzen: Domowina-Verlag.

Kotтhoff H., Nübling D., 2018, Genderlinguistik. Eine Einführung in Sprache, Gespräch und Geschlecht, Tübingen: Narr.

Leiss E., 1994, Genus und Sexus. Kritische Anmerkungen zur Sexualisierung von Grammatik, in: Linguistische Berichte 152, S. 281-300.

Lobin H., 2021, Sprachkampf. Wie die Neue Rechte die deutsche Sprache instrumentalisiert, Berlin: Dudenverlag.

Menzel Th., 2021, Genderlinguistik des Sorbischen: Flexionsmorphologische Grundlagen, in: Lětopis 68/2, S. 105-135.

Menzel Th., Pohontsch A., 2020, Sorbisch, in: Plewnia A., Beyer R. (Hgg.), Handbuch der Sprachminderheiten in Deutschland, Tübingen: Narr, S. 229-271.

Motschenbacher H., 2015, Structural gender linguistics and de-essentialisation. A study of Croatian personal nouns, in: Scheller-Boltz D. (Hg.), New approaches to gender and queer research in Slavonic studies, Wiesbaden: Harrassowitz, S. 51-69. 
NeKVAPIL J., 2007, On the relationship between small and large Slavic languages, in: International journal of the sociology of language 181, S. 141-160.

Šatava L., 2005, Sprachverhalten und ethnische Identität. Sorbische Schüler an der Jahrtausendwende. Bautzen: Domowina-Verlag (= Schriften des Sorbischen Instituts; 39).

Scheller-Boltz D., 2015, From isolation to integration. Gender and queer research in Slavonic linguistics: Challenges, approaches, perspectives. An introduction, in: (ders., Hg.), New approaches to gender and queer research in Slavonic studies, Wiesbaden: Harrassowitz, S. 15-31.

Wąsowska A., 2020, Wszyscy równi wobec języka! [online:] http://wasowska.net/ jezyk-neutralny-plciowo/, [19.05.2021].

\section{"Gender-Equal Speech" as Evidence for Language Contact: The Case of Sorbian ( s u m mary)}

In a small corpus study with texts from Lower and Upper Sorbian newspapers, we examined the distribution of linguistic means of gender equity in Sorbian. By means of the close-reading method, several strategies of splitting (co-naming) and gender neutral constructions were considered. In general, explicit gender-linguistic structures such as co-naming are not very common in our texts. They are rather to be understood as rarely used emblematic linguistic symbols. The generic masculine, instead, is widespread in the texts. Lexically, the linguistic means of gender-equity are easily recognisable as adoptions from German.

Keywords: Sorbian; gender linguistics; language contact; minority languages; newspaper language 
